\title{
ANTISACCADES AND EXECUTIVE CONTROL
}

\author{
Adam TARNOWSKI \\ Military Institute of Aviation Medicine, Department of Psychology, Warsaw, Poland
}

Source of support: B+R funds, Task XIII /7.

Author's address: A. Tarnowski, Military Institute of Aviation Medicine, Department of Psychology, Krasińskiego 54/56 Street, 01-755 Warsaw, Poland, e-mail: tarnowski@wiml.waw.pl

Introduction: Directing the eye gaze to the opposite side when a stimulus appears, demands inhibition of natural reflex and intentional control when performing the movement. Under normal conditions, the reflexive saccades are guided by attention map, combination of visual saliency and task relevance. In the antisaccade task a conflict between task relevance and saliency maps must be resolved, thus the problem involves the central executive resources.

Methods: The study involved 26 individuals, 12 men and 14 women. The saccadic reaction times were measured in trials involving 5 different verbal memory workload levels.

Results: Results have shown only a weak effect of memory workload, the latencies were longer when memory was not overloaded. Also, the proportion of saccades in the wrong (toward the stimuli) direction remained nearly constant when the memory workload increased.

Discussion: The experiment results suggest that the introduction of a memory task reduces the executive resources involved in the antitask.

Conclusions: It is difficult to maintain attention and gaze on a fixation point for a few seconds between the stimuli. In a dual-task paradigm, increasing the difficulty of one task does not necessarily increase the processing time of the second task.

Keywords: antisaccades, saccadometry

Figures: 3 • References: 14 • Full-text PDF: http://www.pjamp.com • Copyright C 2013 Polish Aviation Medicine Society, ul. Krasińskiego 54/56, 01-755 Warsaw, license WIML • Indexation: Index Copernicus, Polish Ministry of Science and Higher Education 


\section{INTRODUCTION}

There are reasons supporting the opinion that the antisaccade task is one of the most important tools in the eye movement research. After over a century of eye movement research, it becomes clear that oculomotor studies open the gate to understanding the mind and the brain. For psychologists investigating the relationship between automated and controlled behavior, there is no other human activity where such interactions could be observed in a better and easier way. Among other tests used in oculomotor research, the antisaccades are engaging most of the "central", "voluntary" and "executive" resources of the human cognitive system. Tasks involving higher mental functions, such as memory, problem solving and thinking are very sensitive to distractions and may depend on the subjective experience, thus becoming an important source of error. The control of antisaccades demands a great deal of "executive resources", it is therefore considered to be a promising tool for the study of higher mental functions.

When a task demands conscious attention and executive resources, the natural question is the role of working memory in saccade generation. More than a decade before WM model was proposed, Saul Sternberg had published his famous results and launched a revolution in the chronometric investigation of higher cognitive functions [12]. In summary, he discovered the effect of linear increase of reaction time when subjects were asked to remember an increasing number of elements. This effect, well-proven in multiple reproductions, became a clear criterion of the engagement of central resources in cognitive tasks. Consideration of the antisaccade task as a model of controlled cognitive activity exploring its relation with working memory appears as an important issue.

Researchers, who wish to use antisaccades in the studies of the human behavior, must resolve a serious dilemma. The research should be "environmentally valid". Tasks performed in the laboratory should closely match the natural activities, as much as possible. There are still very few studies with good-quality eye movements recorded in a natural environment. Antisaccade researchers must face even more difficult challenges, because the antisaccade is not a natural activity at all.

The antisaccade task (or simply "antitask") has been developed by Peter Hallet [5]. Its essential element involves moving the gaze to the opposite side in relation to the stimulus displacement. More general consideration is required to integrate this classical setup with the current knowledge about visual attention.
When moving the eyes, we should determine where the most crucial information within the visual field is located and when the movement should be executed [4]. Under normal (prosaccadic) conditions, the destination point for eye movement is determined by the bottom-up and topdown processes. Reflexive eye movements to the novel or physically attractive stimuli are guided by the saliency map [6]. Saliency map represents the visual attractiveness of each object present in the visual field. It is possible that saliency depends on the primary characteristics of the object: the color, intensity, line orientation and movement. In the reflexive, bottom-up mode, saccade goes toward an object of maximum salience.

Controlled, task-dependent eye movements are mostly directed by the task-relevance map, based on the task representation in the working memory [10]. The working memory processes the actual task. The task determines the information required, its logical analyses and the experience already possessed, then prompts the areas of interest. In the top-down mode, the saccade is guided to the most important object. The attention map, which determines the direction of movement, is combined with saliency and task relevance map. Dynamics of the attention map should take into consideration the effects of decreasing novelty of the existing stimuli, the development of novel objects, modification of information requirements resulting from task planning and its monitoring, the narration of observed actions and, last but not least, the inhibition of return phenomena.

Perceptual space is not geometric, rather it is described by a system of coordinates. We recognize gestalts even before they can be identified as objects [1]. Human attention is not guided by the Cartesian coordinates, but rather it shifts between objects. Mortier, Donk and Theeuwes have shown that when an object is cued, there are more benefits for attention allocation when it is guided into the objects rather than into locations [9].

Therefore, once it is determined where the gaze should be directed, there remains the problem of when it should proceed. In most of our (prosaccadic and antisaccadic) experiments, the answer to the question of "when" is very simple - we instruct the subjects to move their eyes as fast as possible once the stimulus appears. In the real world such reactions are unique. Most saccades are directed towards the objects represented on the attention map, only few of them are the reactions to novel stimuli. When a new salient object appears, it captures our attention and gaze, but among millions 
of saccades performed, only a small percentage is guided this way. Usually, we look at something interesting within our visual field once the cognitive exploration of an object in the previous fixation location becomes terminated. The time course of eye movements is more dependent on the "release" and disengagement of attention from the previous fixation, thus allowing for an assignment on a new stimulus [3]. An ideal subject in oculomotor experiment fixates on the center of the visual field for a few seconds (if nothing interesting is found, a static point is displayed) and then reacts to the external stimulus. Therefore, both the fixation and the target's gradual jerks are far from natural conditions. It can be concluded, that in the reality of such experiment, the subject's task is primarily the inhibition of visual exploration leading to a form of visual deprivation.

In summary, directing the eye gaze is a complex mechanism in which the mind must solve it 2 or 3 times in every second. Oftentimes it is not possible to control it in a serial and ordered manner. On the other hand, the eye movement mechanisms must be programmable in a way to spare the central executive resources, as much as possible.

Antisaccade tasks involve all features present in a classical experiment and add a few new challenges. The expected eye movements should be directed "nowhere", it is to a location with zero saliency, to an empty space in the visual field, opposite and equally distant to the flashing object. Following such instructions is difficult for many individuals, especially children.

The unnatural character of antisaccade task is both an advantage and a potential disadvantage. The experiments have poor "ecological validity", but the engagement of executive control with occulomotor activity is effectively forced. Research has proven that performance of antisaccade task is linked to executive functions. Unsworth, Schrock \& Engle have found a correlation between antitask performance and the capacity of working memory [14]. Very well documented poor antitask performance found in schizophrenia and dyslexia (see Everling, Fischer for review) also proves a connection between the ability to voluntarily move the gaze and the clinical problems with self-control [2]. A researcher must carefully control the conditions, because any deviation from the usual mechanism of attention control may be the source of an artifact. According to the authors, the restrictions set by the disadvantages of the antisaccadic task may be overcome by a slight modification of antisaccade task. In the experiment presented below, two additional frames were displayed con- tinuously on the screen. When stimulus was presented inside one of the frames, the subjects were asked to move their gaze into the opposite frame.

Empirical data concerning antisaccade task subjected to various distractors are available. Kloft, Reuter, Viswanathan, Kathmann and Barton have recently found a robust positive effect of symbolic cueing antisaccade target on the reaction time [7]. Similar observations have been reported earlier by Roberts, Hager and Heron [11]. Mitchell, Maccrae and Gilchrist observed the same effect [8]. They concluded their experiments with an important suggestion, in their opinion, the blocking reflexive movement toward the stimulus is impeded by a working memory task that is stronger than that of guiding the eye into a new and opposite location.

The relationship between working memory and prosaccades (saccadic eye movements towards the stimulus) has been explored in previous research and lead to an interesting finding: as the verbal memory workload was increasing, the saccadic reaction time was decreasing in a linear fashion [13]. This effect, opposite of the classic Sternberg rule, provided evidence that increasing the engagement of the central resources in memory task had forced a kind of automation in saccades control.

The experiment described below attempted to replicate this effect in the antisaccade task. It is hypothesized that increasing memory load will engage central executive resources. As a result, the effect on reaction time of intentionally guided antissaccacades should be observed.

\section{METHODS}

\section{Subjects}

The study involved 26 individuals, 12 men and 14 women, students of the Faculty of Psychology, University of Warsaw, and aged 19 to 29 years. According to the provided declarations, the subjects were generally healthy.

\section{Apparatus and stimuli}

Measurements were made with Ober2 Oculograph and a computer generating the stimuli. Chin rest was used to immobilize the head at a distance of $50 \mathrm{~cm}$ from the monitor screen. Sets of $1,3,5$ and 7 letters were presented before the beginning of each series. One of the series has not used a memory task, only the recording of eye movement responses. Visual stimulation program began after the letter sets have been memorized. Subjects were asked to look directly at a cross located in the middle of the screen (Fig. 1.) until 


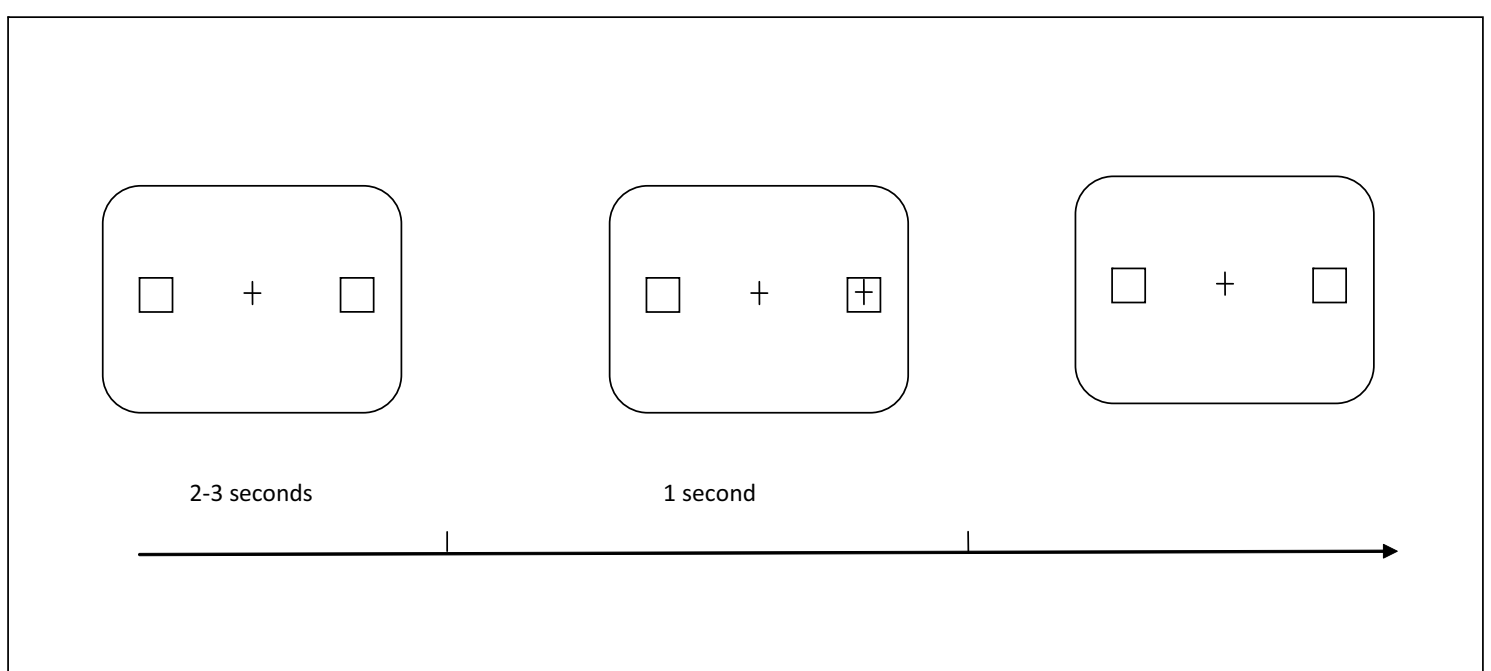

Fig. 1. Stimuli presentation scheme in experiment.

a stimulus appeared in one of the squares. The squares were located within a 10-degree angle from the point of initial fixation.

A cross was presented in one of the squares at random intervals (every 2-3 seconds). The subject's task was to gaze at the opposite square. Each stimulus was present for 1 second. A total of 40 stimuli were presented in each of the series.

\section{Procedure}

Each of the subjects participated in 5 series of exposures, differing only by the memory load. The order of the series was modified in accordance with the principle of Latin square. Each series last- ed about 100 seconds. Duration of the study (including instructions and exercises) did not exceed 10 minutes. Subjects received a small gift, but no salary, for their participation in the study.

\section{RESULTS}

Saccadic reaction time and the proportion of eye movements in the wrong direction have been used as indicators of speed and accuracy of the task. According to the hypothesis, the saccadic reaction time decreases with increasing memory load. The results are presented in Fig. 2. As predicted, decrease in the response time was

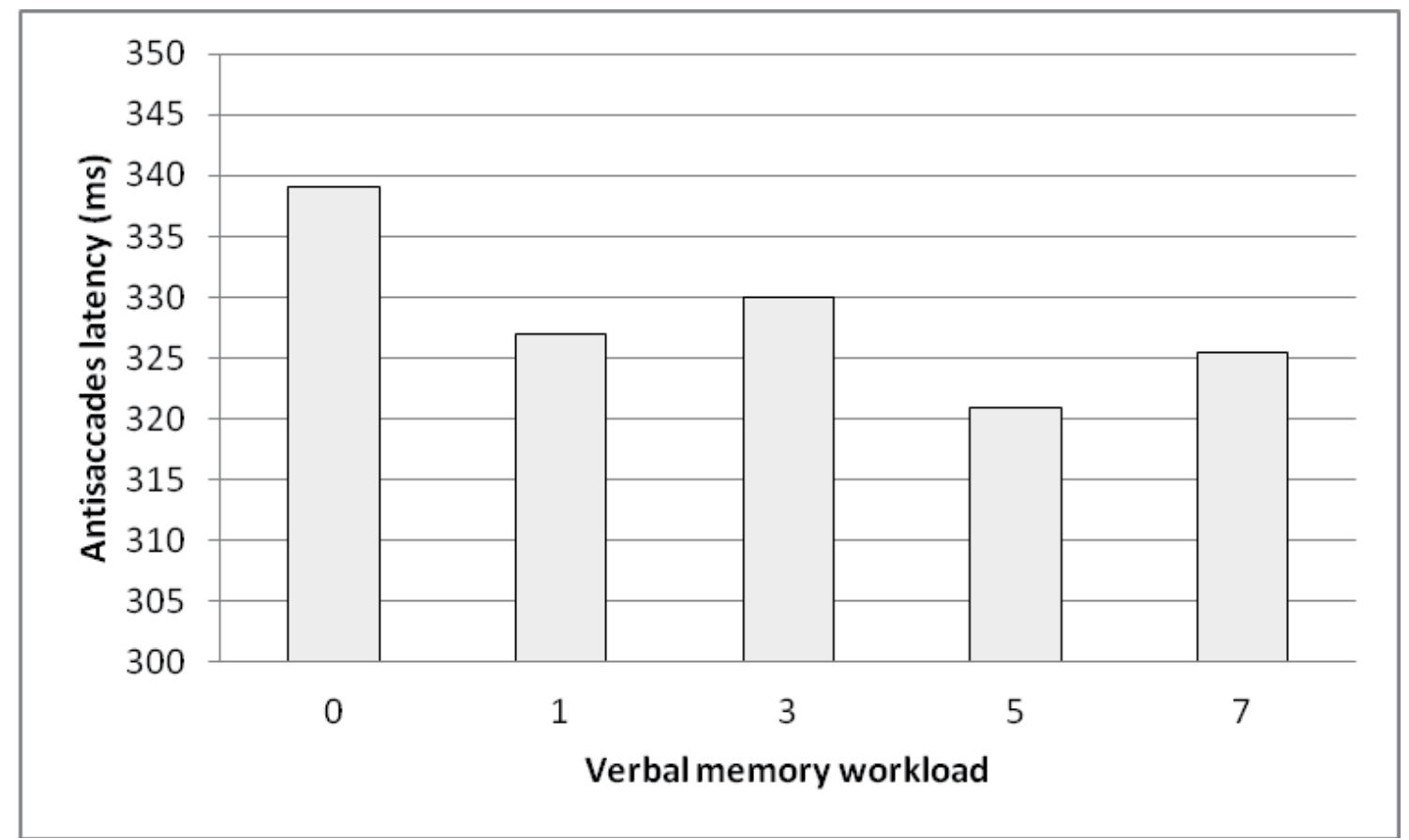

Fig. 2. Stimuli presentation scheme in experiment. 


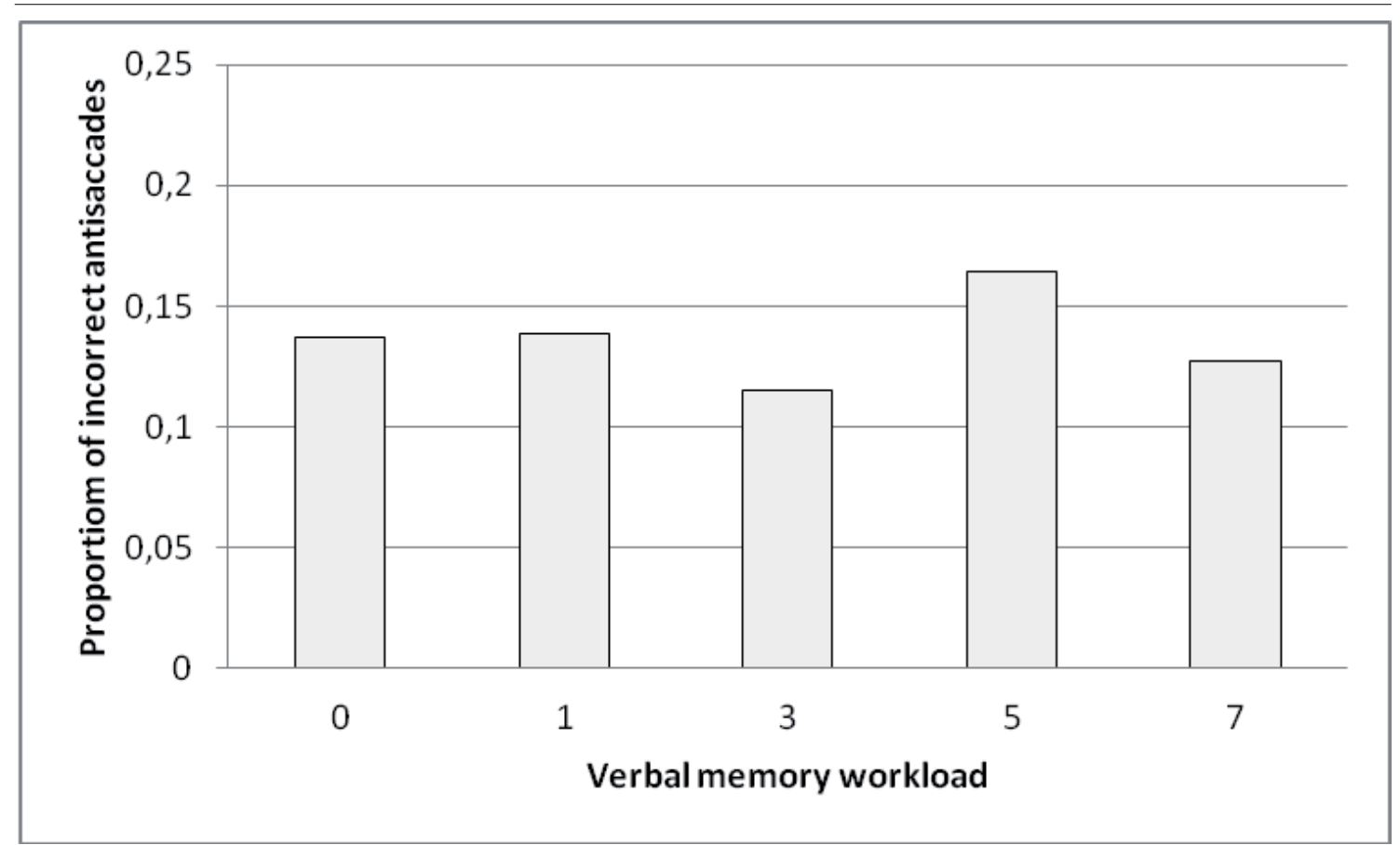

Fig. 3. Dependence between memory workload and proportion of incorrect antisaccades.

statistically significant $(F[4.100]=3.05, p=0.020$, $\left.\eta^{2}=0,10\right)$, but the correlation was not linear. Reaction times decreased abruptly after the memory task and then remained at a similar level.

Hypothesis concerning increasing automation of eye movement control (an increase in the number of responses in the wrong direction) has not been confirmed. Importantly, there were fewer incorrect antisaccades under neutral conditions without memory workload. The proportion of eye movement in the wrong direction is presented in Fig. 3. The effect is not statistically significant $(F[4 ; 100]=1.78, p>0.1)$.

It should also be mentioned, that 9 of the subjects were unable to recall 7 letters after completing the experiment.

\section{DISCUSSION}

The experiment results suggest that the introduction of a memory task reduces the executive resources involved in the antitask. According to Findlay and Walker, it causes a weaker intentional control of cognitive processing, decreased activation of central fixation and shorter response times to target movements [4].

Another possible explanation is that the higher-level control of saccadic activity has been advantageous for the simultaneously performed memory task. Considering such an alternative one can expect an increased number of erroneous responses due to inattention. The author has not observed any increase in erroneous responses. This could possibly be associated with a better description of the expected saccadic behavior to a clearly defined anti-target gaze location (gaze allocation frame).

It should be stressed that the observed effect is contrary to some data published in the past $[7,8,11]$. Obviously, when any two processes are competing for the same resource, slower reaction times are to be expected. However, in presented study the memory task required no actual activity, the elements memorized were passively kept in mind, so there was no competition for the executive resources.

The modified antitask is relatively simple, because it only consists of inhibition of reflexive responses, while the implementation of alternative actions relays on the reflex mechanisms based on the saliency map. The engagement of executive control for evoking antisaccades has an impact on the process of recalling memory. A set of 7 letters to recall is below what would be expected from a group of university students.

\section{CONCLUSIONS}

Antisaccade task is not natural for humans. It is difficult to maintain attention and gaze on a fixation point for a few seconds between the stimuli. More natural antisaccades can be observed in a setup with 2 frames presented on the screen. 
In a dual-task paradigm, increasing the difficulty of one task does not necessarily increase the processing time of the second task. Resources engaged in the primary task may call for automa- tion of the other task and lead to a decrease of its reaction time, either with or without affecting the accuracy of the response.

\section{AUTHORS' DECLARATION:}

Study Design: Adam Tarnowski; Data Collection: Adam Tarnowski; Statistical Analysis: Adam Tarnowski; Manuscript Preparation: Adam Tarnowski; Funds Collection: Adam Tarnowski. The Author declares that there is no conflict of interest.

\section{REFERENCES}

1. Driver J, Baylis GC: Attention and visual object segmentation. In: Parasuraman R ed. The attentive brain. Cambridge: MIT Press; 1998:299-325.

2. Everling S, Fischer B: The antisaccade: a review of basic research and clinical studies. Neuropsychologia 1998; 36:88599.

3. Findlay JM, Gilchrist ID. Active Vision: The Psychology of Looking and Seeing. Oxford: Oxford University Press; 2003.

4. Findlay JM, Walker R. A model of saccade generation based on parallel processing and competitive inhibition. Behavioral and Brain Sciences 1999; 22:661-74.

5. Hallett PE. Primary and secondary saccades to goals defined by instructions. Vision Research 1978; 18:1279-96.

6. Itti L, Koch Ch. Computational modeling of visual attention. Nature Reviews-Neuroscience 2001; 2:194-203.

7. Kloft L, Reuter B, Viswanathan J, Kathmann N \& Barton JJS. Response selection in prosaccades, antisaccades, and other volitional saccades. Exp Brain Res 2012; 222:345-53.

8. Mitchell JP, Macrae CN, Gilchrist ID. Working Memory and the Suppression of Reflexive Saccades. Journal of Cognitive Neuroscience 2002; 14: 95-103.

9. Mortier K, Donk M, Theeuwes, J. Attentional capture within and between objects. Acta Psychologica 2003; 113:133-45.

10. Navalpakkam V, Itti L. Modeling the influence of task on attention, Vision Research 2005; 45:205-31.

11. Roberts RJ, Hager LD, Heron C. Prefrontal cognitive processes: Working memory and inhibition in the antisaccade task. Journal of Experimental Psychology General 123 1994:374-93.

12. Sternberg S. High-speed scanning in human memory. Science 1966; 153:652-54.

13. Tarnowski A. Effect of increasing verbal and visuospatial memory workload on saccadic eye-movement latency. Perception 2007; 36 supplement:35.

14. Unsworth N, Schrock JC, Engle RW. Working memory capacity and the antisaccade task: individual differences in voluntary saccade control. Journal of Experimental Psychology: Learning, Memory, and Cognition 2004; 30:1302-21.

Cite this article as: Tarnowski A. Antisaccades and Executive Control. Pol J Aviat Med Psychol, 2013; 19(4): 5-10. 